\title{
Der Arbeitskreis Geologie und Geophysik der Polargebiete
}

\author{
Jens O. Herrle ${ }^{1}$, Cornelia Spiegel ${ }^{2}$, Andreas Läufer ${ }^{3}$, and Jean-Pierre Paul de Vera ${ }^{4}$ \\ ${ }^{1}$ Institut für Geowissenschaften, Goethe-University Frankfurt, Altenhöferallee 1, 60438 Frankfurt am Main, Deutschland \\ ${ }^{2}$ Institut für Geowissenschaften, Universität Bremen, Postfach 330440, 28334 Bremen, Deutschland \\ ${ }^{3}$ Bundesanstalt für Geowissenschaften und Rohstoffe, Postfach 510153, 30631 Hannover, Deutschland \\ ${ }^{4}$ Deutsches Zentrum für Luft- und Raumfahrt e.V. (DLR), Raumflugbetrieb und Astronautentraining Microgravity User \\ Support Center (MUSC), Geb. 29, Linder Höhe, 51147 Köln, Deutschland
}

Correspondence: Jens O. Herrle (jens.herrle@em.uni-frankfurt.de)

Published: 20 May 2021

\section{Rückblick der AK Geologie und Geophysik der Polargebiete}

Die geologischen und geophysikalischen Arbeiten in den Polargebieten und Eisregionen der Hochgebirge bilden eine zentrale Komponente der Erforschung des Systems Erde im Spannungsfeld zwischen der Rekonstruktion des Klimawandels auf geologischen Zeitskalen, der Entdeckung und Evaluation von Ressourcen, bis hin zum Testfeld für zukünftige Raumfahrtforschung. Der besondere Reiz liegt aber sicherlich an der einmaligen Kombination von fundierter Forschung, ihren immer noch großen Unwägbarkeiten vor Ort durch Wetter, schwierigem Gelände, komplizierter Logistik, der Abgeschiedenheit und des geteilten Lebens auf engsten Raum. Es hat sich damit seit den großen Entdeckungsreisen zu den Polarregionen des 19. Jahrhunderts nichts an diesem vielfältigen und verführerischen Charme geändert, welchem auch die heutigen Forschenden in den Polargebieten und Hochgebirgen immer noch unterliegen. Einem Charme, der sich durch die unterschiedlichsten Charaktere der Forschenden bei den jährlichen Treffen des Arbeitskreises Geologie und Geophysik der Polargebiete widerspiegelt und diese wissenschaftlich und menschlich sehr interessant macht.

Der Arbeitskreis Geologie und Geophysik der Polargebiete ging aus dem ehemaligen Arbeitskreis Geologie der Polargebiete hervor, welcher 1983 auf eine Initiative von Dieter K. Fütterer und Georg Kleinschmidt ins Leben gerufen worden ist. Erste Sprecher und Organisatoren dieses Arbeitskreises waren Dieter K. Fütterer und Hubert Miller (beide 1983-1993) gefolgt von Hans-Wolfgang Hubberten und Hans-Jürgen Paech (beide 1993-2005). 2005 wurde der Arbeitskreis in „Geologie und Geophysik der Polargebiete“ umbenannt und bis 2017 durch Martin Melles und Solveig Estrada erfolgreich geleitet (Melles und Estrada, 2009). Seit 2018 sind Jens O. Herrle und Cornelia Spiegel die Sprecher*innen des Arbeitskreises und organisieren die jährlichen Treffen an wechselnden Orten. Dabei kommen Arbeitskreismitglieder aus einer Vielzahl an Universitäten und polarforschenden Institutionen wie das Alfred Wegener Institut - Helmholtz-Zentrum für Polar- und Meeresforschung (AWI), die Bundesanstalt für Geowissenschaften und Rohstoffe (BGR) und das Deutsche Zentrum für Luft- und Raumfahrt (DLR) zusammen, um Informationen über laufende Projekte und Projektideen auszutauschen und Zukunftspläne für den Arbeitskreis und für Forschungsvorhaben zu entwickeln. Ein wichtiges Instrument der Forschungsförderung ist dabei das Schwerpunktprogramm 1158 ,Antarktisforschung - mit vergleichenden Untersuchungen in arktischen Eisgebieten" der Deutschen Forschungsgemeinschaft.

Seit der Gründung des Arbeitskreises wurden vier verschiedene Strategieschriften zur Ausrichtung und Relevanz der Polarforschung verfasst, welche die Entwicklung der deutschen Polarforschung und Ausrichtung im Nachhinein exemplarisch aufzeigen. Strategieschrift 1 priorisierte geologische Forschungsprogramme in der Antarktis mit Schwerpunkten zur Landarbeit und dem Weddellmeer (1984-1989). Die Strategieschrift 2 konzentrierte sich auf Arbeiten arktischer Meeresräume (1984). Strategieschrift 3 befasste sich mit der geowissenschaftlichen Polarforschung in ihrer ganzen Breite insbesondere zu Klimauntersuchungen in den Polarregionen und wissenschaftlichen Tiefbohrungen (1999). Mit der Änderung der Namensgebung des Arbeitskreises 2005 wurde die zunehmende Vernetzung der beiden geowissenschaftlichen Teildisziplinen der Geologie und der Geo- 
physik in der Polarforschung hervorgehoben. Dies spiegelt sich vor allem in der Strategieschrift 4 des Arbeitskreises wider, welcher durch das Engagement von Martin Melles und Solveig Estrada sowie den Mitgliedern des Arbeitskreises entstanden ist und 2015 in der Zeitschrift Polarforschung publiziert wurde (Melles et al., 2015). Die Strategieschrift bietet ein informatives aperçu über den status quo der geowissenschaftlichen Polarforschung und ihrer gesellschaftlichen und politischen Bedeutung. Sie wirft wichtige Schlüsselfragen auf, die in den kommenden Jahren bezüglich der Geologie des Untergrundes und der Rolle der Polargebiete im globalen Klimasystem beantwortet werden müssen, um Veränderungen in den polaren Regionen und ihre Rückkopplungen im System Erde besser vorhersagen zu können. Damit bietet die Schrift einen wichtigen Leitfaden für die deutsche Polarforschung. Neben diesen Schriften bildet der 2017 erschienene DFG-Statusbericht des Deutschen Nationalkomitees Scientific Committee on Antarctic Research (SCAR) und International Arctic Science Committee (IASC) „Polarforschungsagenda 2030 Status und Perspektiven der deutschen Polarforschung" unter der Leitung von Günther Heinemann ebenfalls einen wichtigen Beitrag zur Forschung in den Polargebieten (Heinemann et al., 2017). Auch an der Erstellung dieser Schrift haben Mitglieder des Arbeitskreises aktiv mitgewirkt. Die Agenda gibt einen sehr guten Überblick über die Geschichte und Organisation der deutschen Polarforschung und zu zukünftigen Forschungsstrategien der DFG, die Themen rund um die Erforschung der Polargebiete im globalen Wandel, im System Erde, in Fragen nach Ressourcen und Nachhaltigkeit sowie deren Umsetzung fokussiert.

In den letzten Dekaden sind die Institutionen des AWIs und der BGR das wesentliche Rückgrat des Arbeitskreises Geologie und Geophysik der Polargebiete. Die beiden Institutionen bilden sowohl wissenschaftlich als auch durch ihre logistische Unterstützung in Deutschland ein zentrales Bindeglied zu den Universitäten, mit denen gemeinsame Projekte, Ausfahrten oder Landexpeditionen durchgeführt werden. Die Polarforschung der BGR kann dabei auf eine über 40-jährige Geschichte zurückblicken. Die Forschungsarbeiten der BGR legen den Schwerpunkt auf die Krustenstruktur und geologisch-tektonische Entwicklung des antarktischen Kontinents sowie der zirkum-arktischen Landmassen und der Öffnungsgeschichte des Nordpolarmeeres. Die geologischen Landarbeiten werden in der Regel durch aerogeophysikalische Befliegungen sowie geophysikalische marine Messfahrten ergänzt. Das seit Anfang der 1990er Jahre durchgeführte CASE-Programm (Circum-Arctic Structural Events) untersucht die dynamisch-strukturelle Entwicklung der Landbereiche rund um das Nordpolarmeer. Es hat sich mittlerweile zu einer international anerkannten Forschungsplattform entwickelt, die es zahlreichen Forschenden von Regierungsdiensten und Universitäten möglich macht, multidisziplinäre geowissenschaftliche Studien und Geländearbeiten in einem sehr entlegenen Teil unseres Planeten durchführen zu kön- nen. Eine Vielzahl der wissenschaftlichen Ergebnisse des CASE-Programms sind jüngst anlässlich dessen 25-jährigen Bestehens in einem Sonderband der Geological Society of America herausgegeben worden (Piepjohn et al., 2019). Das Transantarktische Gebirge des Victoria-Landes und seine Nachbarregionen sind das Zielgebiet des seit 1979 durchgeführten GANOVEX-Programms (German Antarctic North Victoria Land Expedition). Zu dessen logistischer Unterstützung betreibt die BGR die Gondwana-Station an der TerraNova-Bucht und unterhält die Lillie-Marleen-Hütte im Transantarktischen Gebirge. Das GANOVEX-Programm wurde durch Franz Tessensohn initiiert und markiert den Beginn der festlandsgeologischen Forschungsarbeiten der BGR in den Polargebieten. Als international anerkanntes und multidiszplinär ausgerichtetes geowissenschaftliches Forschungsprogramm ist es heute als „Markenname“ in der Antarktisforschung in der Rossmeerregion angesehen. So fanden Mitglieder des Arbeitskreises Hinweise darauf, dass die Gesteine des Transantarktischen Gebirges von bis zu vier Kilometer mächtigen jurassischen bis paläogenen Sedimenten überlagert waren. Diese Sedimente wurden mit der Heraushebung des Gebirges vollständig abgetragen und finden sich heute als Abtragungsschutt in den Becken des Rossmeeres. Das Programm GEA (Geodynamic Evolution of East Antarctica) wird seit 2010 mit bisher fünf geologisch-geophysikalischen Kampagnen in enger Kooperation mit dem AWI durchgeführt. Es wurde aus mehreren in der Ostantarktis angesiedelten Vorläufer-Projekten entwickelt, zu denen insbesondere auch das unter maßgeblicher Beteiligung der BGR durchgeführte AGAP-Projekt (Antarctica's Gamburtsev Province) als eines der Flaggschiff-Programme des Internationalen Polarjahres (IPY) 2007-2009 zählte. Die bisherigen Ergebnisse des GEA-Projekts haben zu einem völlig neuen Bild der Subeis-Geologie der Ostantarktis geführt, so zum Beispiel die Entdeckung des mindestens $500000 \mathrm{~km}^{2}$ großen Tonian Arc Super Terrane (TOAST) als bedeutenden, an der Bildung Gondwanas beteiligten Krustenfragment. Die BGR war maßgeblich an den internationalen Bohrprogrammen Cape-Roberts-Project und dessen Folgeprojekt ANDRILL im Rossmeer beteiligt. Deren Resultate lieferten eine durchgehende Klimakurve für den Zeitraum der letzten etwa 25 Millionen Jahren bis heute.

Das AWI nimmt eine zentrale Position in der deutschen Polarforschung in seiner ganzen Breite ein, da sie Forschungsschiffe wie die Polarstern, Heincke, Uthörn und Mya II, zwei Flugzeuge und die Forschungsstationen NeumayerStation III und Kohnen-Station in der Antarktis, die deutschfranzösische AWIPEV-Station auf Spitzbergen, die russischdeutsche Forschungsbasis Samoylov im Lenadelta, das Dallmann Laboratory auf dem King George Island und das Drescher Ice Camp auf dem Riiser-Larsen Schelfeis betreibt. Seit der Gründung des AWIs 1980 im Zuge des deutschen Beitritts zum Antarktisvertrag liegt der Forschungsschwerpunkt des AWIs auf der Erkundung der Geologie, Struktur, Erdgeschichte der Polarregionen sowie der Klimaforschung, 
Meeresbiologie und Meeresgeologie. Die wissenschaftliche und logistische Bedeutung des AWIs kulminiert insbesondere in der im Herbst 2019 gestarteten internationalen MOSAiC Expedition (Multidisciplinary drifting Observatory for the Study of Arctic Climate) mit dem deutschen Forschungseisbrecher Polarstern unter der Leitung von Markus Rex. In diesem für die Polarforschung zur Zeit sicherlich größtem Projekt driftet die Polarstern eingefroren im Meereis durch das Nordpolarmeer, um den Einfluss der Arktis auf das globale Klima besser verstehen zu können.

An den Universitäten wurde in den letzten Jahren die Polarforschung neben vielen einzelnen kleineren Projekten in der kanadischen und russischen Arktis, Spitzbergen und der Antarktis im Wesentlichen durch das wissenschaftliche Bohren des International Continental Scientific Drilling Program (ICDP, seit 1990) im Kratersee El'gygytgyn in Tuschukota in Sibirien unter der Federführung von Martin Melles im Jahr 2008 bestimmt. Die ungestörten Sedimentabfolgen erlauben es Klimaschwankungen, vor allem Niederschläge, Vegetation und Temperatur zeitlich hochaufgelöst in den letzten 3,6 Millionen Jahre zu rekonstruieren. Eine zeitliche Auflösung die sonst nur durch Eiskernbohrungen erreicht wird (Melles et al., 2012). Auch das Integrated Ocean Discovery Program (IODP) setzte in den letzten Jahren einen wesentlichen Schwerpunkt auf die erdgeschichtliche und klimatische Entwicklung und Ozeanographie der Antarktis und Arktis mit mehr als neun wichtigen Bohrfahrten wie unter anderem der Wilkes Land Glacial History Expedition (Exp. 318 2010), Antarctic Paleoclimate and Ice History from George V Land and Adélie Land Shelf Sediments Expedition (Exp. 373, 2016), Amundsen Sea West Antarctic Ice Sheet History Expedition (Exp. 379, 2019), Iceberg Alley and Subantarctic Ice and Ocean Dynamics Expedition (Exp. 382, 2019), und der Arctic Coring Expedition (ACEX) (Exp. 302, 2004). Ein erdgeschichtlicher Glanzpunkt wurde 2017 u. a. mit dem von der Polarstern aus gesteuerten MARUM-MeBo70Meeresbodenbohrgerät in $1000 \mathrm{~m}$ Wassertiefe bei etwa $73^{\circ} \mathrm{S}$ $\left(82^{\circ} \mathrm{S}\right.$ vor $\left.90 \mathrm{Ma}\right)$ auf dem westantarktischen Schelf in der Pine Island Bay erbohrt. Die Bohrung erbrachte kurz vor Abbruch auf den letzten Bohrmetern ein fossiles Wurzelnetzwerk in einem pollen- und sporen-beladenen Tonstein hervor. Ein Zufallsfund, welcher aber von zentraler Bedeutung für die Entwicklung der Antarktis ist, und der belegt, dass während der mittleren Kreide die Antarktis eine gemäßigte Regenwaldvegetation aufwies (Klages et al., 2020). Bedingungen, die nur unter hohen atmosphärischen $\mathrm{CO}_{2}$ Konzentrationen (1120-1680 ppm) existieren. Dieser Fund kombiniert mit einer Klimamodellierung erlaubt es, Modelle zu kalibrieren und zu präzisieren, was für die Simulation eines potentiell zukünftigen Hoch- $\mathrm{CO}_{2}$-Klimas von großer Wichtigkeit ist. Einen sehr guten und aktuellen Überblick geologischer Forschung in der Antarktis gibt das von Georg Kleinschmidt als Editor und Autor verfasste Buch "The Geology of the Antarctic Continent" (Kleinschmidt, 2021), mit vielen
Beiträgen internationaler Wissenschaftler*innen und starker Beteiligung unserer Mitglieder aus dem Arbeitskreis.

Darüber hinaus zeigt sich die Breite des Arbeitskreises an der Relevanz der Polarregionen für planeten-analoge Feldstudien. Im Rahmen der Helmholtz-Allianz „Planetary Evolution and Life (PEL, 2009-2013)“" und einem Weltraumexperiment BIOMEX (das biologische Marsexperiment, des Deutschen Zentrums für Luft- und Raumfahrt [DLR] 20102019) wurden im Rahmen des GANOVEX-Programms der BGR Feldstudien im Nord-Victoria-Land durchgeführt, die die Aufgabe hatten, planeten-analoge Gebiete mit MarsRelevanz aufzuspüren.

In allen oben beschriebenen Projekten waren Mitglieder des Arbeitskreises involviert. Es ist eine Herausforderung, die vielfältigen Forschungsvorhaben und Interessen in einem kurzen Artikel zusammenzufassen, und dabei allen gerecht zu werden. Letztlich gibt die 2015 durch unseren Arbeitskreis verfasste Strategieschrift zum Thema „Geowissenschaftliche Polarforschung in Deutschland - globale Bedeutung und Perspektive" neben Schlüsselfragen zur Polarforschung einen sehr guten Überblick zu einzelnen Forschungsthemen und zur Struktur der unterschiedlichsten Institutionen, die sich mit der Polarforschung beschäftigen.

Die spannende Frage bleibt, wie sich der Arbeitskreis Geologie und Geophysik der Polargebiete in Zukunft aufstellen möchte. Ein genereller Konsens besteht darin, verstärkt jüngere, für das Thema brennende Wissenschaftlerinnen und Wissenschaftler für unseren Arbeitskreis zu gewinnen und den Arbeitskreis stärker als bisher in der Öffentlichkeit zu etablieren - nicht zuletzt, um damit gezielt auch interessierte Laien an Polarthemen heran zu führen. Die Ausgestaltung, dass aus Wünschen Wirklichkeit werde, müssen wir gemeinsam mit den drei großen Institutionen der Universitäten, der BGR und dem AWI in der Zukunft angehen.

Als derzeitige Sprecher und derzeitige Sprecherin des Arbeitskreises bedanken wir uns bei unseren Vorgängerinnen und Vorgängern und Mitgliedern herzlichst für das große Engagement seit der Etablierung des Arbeitskreises. Wissenschaftlich bleibt zu hoffen, dass es mit den Polarforschungsprogrammen der DFG, der BGR und des AWI sowie den großen Bohrkampagnen wie ANDRILL in der Antarktis in Zukunft weiter gehen wird. Konkrete Planungen der BGR und des AWI gibt es bereits zu einer Bohrkampagne auf dem Ekström-Schelfeis und, gemeinsam mit Neuseeland, einer Bohrung am Rand des Rossmeeres. Neben der zurzeit laufenden arktischen MOSAiC Expedition ist im Rahmen von der European Consortium for Ocean Research Drilling (ECORD) eine Bohrfahrt ,The Arctic Ocean Paleoceanography“ (IODP Exp. 377) für 2022 geplant. 


\section{Interview mit Prof. Dr. Cornelia Spiegel-Behnke} und Prof. Dr. Jens Herrle

Seit 2018 leiten Prof. Dr. Cornelia Spiegel-Behnke und Prof. Dr. Jens Herrle gemeinsam den Arbeitskreis Geologie und Geophysik der Polargebiete. Warum ihnen besonders der Nachwuchs der Polarforschung am Herzen liegt und was ihre Ziele und Visionen für ihren Arbeitskreis sind, erläutern Frau Spiegel-Behnke und Herr Herrle im Interview.

APECS Germany: Wollten Sie schon immer Polarforscher*in werden? Warum haben Sie sich für die Polarforschung entschieden?

J. Herrle: Ich bin von Haus aus Mikropaläontologe und zunächst einmal lag mein Fokus nicht auf den Polargebieten. Während des Studiums bin ich im Rahmen meines Interesses an der Kreidezeit auf ein Buch von Edwin Kemper „Klima der Kreidezeit“" gestoßen, dass sich unter anderem mit der Arktis im Kontext der klimatischen Entwicklung dieser Periode und deren Kälteeinbrüche während extremer Treibhausbedingungen beschäftigt hat, und seitdem fasziniert mich die Arktis. Aber erst 15 Jahre später dann in meiner Position als Professor habe ich die Gelegenheit bekommen, ein kanadisch-deutsches Forschungsprojekt zu dem Thema zu beginnen und auch selbst dort hinzureisen und zu forschen.

C. Spiegel-Behnke: Ich bin lustigerweise nominell Polarforscherin geworden, bevor ich tatsächlich Erfahrungen als Polarforscherin hatte. Ich habe in Kiel Geologie und Paläontologie studiert und dann in Tübingen über eine alpine Fragestellung promoviert. Während meiner Postdoc Stelle in Melbourne bin ich das erste Mal mit der Antarktisforschung in Kontakt gekommen, aber habe dort selbst noch nicht dazu geforscht. Das hat sich dann schlagartig geändert, als ich in Bremen die Professur für Geodynamik der Polargebiete angenommen habe. Ich hatte damals noch keine Erfahrung in den Polarforschung, aber fand das Thema wahnsinnig spannend. Seitdem bin ich Polarforscherin. Ich empfinde es jetzt als sehr bereichernd für meine Forschung, auch die klassischen Ansätze der alpinen Geologie zu kennen, da es einen weiteren Blickwinkel ermöglicht.

APECS Germany: Warum setzen Sie sich für Ihren AK ein? Was motiviert Sie?

C. Spiegel-Behnke: Im Vergleich zu anderen Forschungszweigen der Geowissenschaften ist die Vernetzung in der Polarforschung viel wichtiger. Besonders bei der Vorbereitung und Durchführung von Expeditionen braucht man viel mehr Unterstützung von Anderen und man arbeitet oft mit einem viel größeren Personenkreis zusammen. Deshalb sind Arbeitskreise gerade in der Polarforschung besonders wichtig.

J. Herrle: Die DGP befindet sich in einem Spannungsfeld zwischen dem Alfred-Wegener-Institut, der Bundesanstalt für Geowissenschaften und Rohstoffe und den Universitäten. Um diese verschiedenen Gruppen miteinander in Kontakt zu bringen ist die DGP und unser AK ein sehr nützliches Werkzeug. Besonders für die Arbeitsgruppen, die an
Unis oder Instituten arbeiten, die keinen Schwerpunkt in der Polarforschung haben. Und weil in unserem Arbeitskreis die wissenschaftliche Breite wirklich sehr groß ist, zum einen im Fachlichen von Geologen, über Paläontologen zu Geophysikern, aber auch in den Arbeitsschwerpunkt von Modellierer*innen zu Experten in Expeditionslogistik, sind unsere AK Treffen immer sehr fruchtbar. Unser Arbeitskreis lebt ganz klar vom Input seiner Mitglieder.

APECS Germany: Warum sind Sie AK Leiter*in geworden und was sind Ihre Ziele für den AK?

J. Herrle: Ich habe dieses Amt übernommen, um auch tiefergreifend bei der Entwicklung der Polargesellschaft mitarbeiten zu können. Ich finde, dass wir in der DGP einige neue Akzente setzen könnten, um ein bisschen neuen Schwung zu bekommen. Ein Beispiel was ich kürzlich mit der stellvertretenden Sprecherin des AK Geschichte der Polarforschung Dr. Frigga Kruse gemeinsam angedacht hatte wäre z.B die Öffnung der DGP hin mehr zur interessierten Öffentlichkeit. Der Tourismus in den Polarregionen nimmt zu und das öffentliche Interesse ist sehr groß. Ich glaube, die DGP könnte sich bei diesem Thema breiter aufstellen, um sichtbarer zu sein. Als Leiter des AKs kann ich dabei eine Schlüsselrolle übernehmen, um solche Veränderungen anzustoßen.

C. Spiegel-Behnke: Durch den AK wird man noch viel deutlicher auf bestehende Ungleichgewichte aufmerksam, die man vielleicht in seiner eigenen Arbeitsgruppe nicht so deutlich wahrnimmt. Dabei wird mir besonders klar, dass wir darauf achten müssen, dass wir genügend Nachwuchs haben, um das Wissen an die zukünftigen Polarforscher*innen weitergegeben zu können. Zurzeit findet gerade ein Generationenwechsel in der Polarforschung statt, da viele der Leute, die die Polarforschungsprogramme in Deutschland aufgebaut haben, jetzt in Rente gehen. Deshalb versuchen wir bei den AK Treffen, die durch die kleine Größe auch recht familiär sind, besonders die jungen Wissenschaftler*innen zu ermutigen, sich in die DGP einzubringen und ihre Projekte vorzustellen. Dabei ist uns auch wichtig klar zu machen, dass bei uns nichts in Stein gemeißelt ist. Wir freuen uns sehr, wenn gerade auch vom Nachwuchs Wünsche an uns herangetragen werden, z.B. wie man die AK Treffen anders gestalten könnte oder was noch im AK fehlt.

J. Herrle: Wir sind gerade dabei auch Konzepte zu entwickeln und anzustoßen, die sich besonders darum bemühen, auch den Nachwuchs anzusprechen. Gerade in Bezug auf die Förderung von jungen Wissenschaftler*innen ist aber auch mehr Funding ein wichtiger Baustein, da wir nur so auch den Nachwuchs in die sehr teure Feldarbeit in den Polargebieten integrieren und Wissen weitergeben können.

APECS Germany: Wenn Sie in die Zukunft schauen, was ist in Ihren Augen die dringendste Frage im Forschungsfeld Ihres Arbeitskreises für die nächsten Jahre?

J. Herrle: Unsere dringendsten Fragen drehen sich um die Auswirkungen des Klimawandels auf die polaren Lebensräume. Und dafür brauchen wir ein tiefgreifendes Prozessver- 
ständnis, das wir für geodynamische Prozesse, die sich auf langen und kurzfristigen Zeitskalen ändern, wie z.B. die Interaktion zwischen Prozessen im Erdmantel, Plattentektonik, Klima und Evolution, nur aus den geologischen Archiven beziehen können. Wir brauchen umfassende Forschungsprojekte, die nicht nur Snapshots, sondern so wie z.B. jetzt MOSAiC, eine umfassende Forschung zulassen.

C. Spiegel-Behnke: Für mich steht auch das Prozessverständnis im Vordergrund. Das faszinierende an den Polarregionen ist, dass wir dort wie unter dem Brennglas das Zusammenspiel des Systems Erde beobachten können. Wir können dort untersuchen, wie die langfristigen, tektonischen Vorgänge mit den klimatischen, ozeanographischen und biotischen Prozessen interagieren. Das Ziel für die nächsten zehn Jahre ist es, mit starken, fachübergreifenden Kooperationen dieses Prozessverständnis zu erweitern.

J. Herrle: Und dazu gehören dann auch sozioökonomischen Fragestellungen für die Menschen, die in den Polargebieten wohnen. Da sehe ich großes Potential in der DGP durch ihre vielen, breitaufgestellten Arbeitskreise.

APECS Germany: Was darf für Sie bei der (Feld-)arbeit nie fehlen?

J. Herrle: Tea (Earl Grey) with a cloud of milk. Und für die Feldarbeit in der Arktis natürlich der Schutz vor Eisbären und zu neugierigen Moschusochsen.

C. Spiegel-Behnke: Also ganz pragmatisch in den Polargebieten: ein Helikopter. Und sonst natürlich mein Hammer, das GPS Gerät und nette Geländepartner*innen.

APECS Germany: Was war für Sie die einprägsamste Erfahrung in der Polarforschung?

J. Herrle: Für mich gibt es kein konkretes Erlebnis, aber was mich immer wieder reizt und in die Arktis zieht, ist die Schönheit, Weite und Abgeschiedenheit dieser Landschaft.

C. Spiegel-Behnke: Meine einprägsamste Erfahrung war sehr ambivalent. Wir waren 2018 in Nordgrönland, ungefähr auf $83^{\circ}$ Nord unterwegs. Wir saßen an einem schönen, sonnigen Tag auf einer Klippe, wahrscheinlich $500 \mathrm{~m}$ über dem Meer. Die Klippe war aus einem hellen Sandstein und wir hatten fast den Eindruck von einem weißen Sandstrand aus auf das blaue Meer zu blicken. Das war ein wunderschöner Anblick, aber wenn man bedenkt, dass wir soweit nördlich waren, war der Anblick des offenen Meeres eigentlich einer, der nicht sein sollte. Das war ein sehr eindrücklicher Moment.

APECS Germany: Vielen Dank für das Gespräch.

Das Gespräch führte: Luisa von Albedyll

Datenverfügbarkeit. Für diesen Artikel wurden keine Datensätze genutzt.

Autorenmitwirkung. JOH und CS entwarfen die vorliegende Arbeit. Alle Autoren haben zum Schreiben beigetragen.
Interessenkonflikt. Die Autor*innen erklären, dass kein Interessenkonflikt besteht.

Danksagung. Wir danken allen ehemaligen Vorsitzenden und Mitgliedern des Arbeitskreises „Geologie und Geophysik“ der Deutschen Gesellschaft der Deutschen Gesellschaft für Polarforschung (DGP) für ihre kontinuierlichen wissenschaftlichen Beiträge und Engagement, sowie dem Alfred-Wegener-Institut, HelmholtzZentrum für Polar- und Meeresforschung (AWI), der Bundesanstalt für Geowissenschaften und Rohstoffe (BGR) und deutschen Universitäten mit umfangreichen Polarforschungsprogrammen für ihre langjährige Unterstützung.

Begutachtung. This paper was edited by Donovan Dennis.

\section{Literatur}

Heinemann, G., Braun, M. Brey, T., Damaske, D., Melles, M., Rhein, M., und Willmes, S. (Hrsg.): Polarforschungsagenda 2030 - Status und Perspektiven der deutschen Polarforschung, Statusbericht des Deutschen Nationalkomitees SCAR/IASC der DFG, 160 Seiten, 2017.

Klages, J. P., Salzmann, U., Bickert, T., Afanasyeva, V., Arndt, J. E., Ebermann, B., Gebhardt C., Hochmuth, K., Küssner, K., Najman, Y., Riefstahl, F., and Scheinert, M.: Temperate rainforests near the South Pole during peak Cretaceous warmth, Nature 580, 81 86, https://doi.org/10.1038/s41586-020-2148-5, 2020.

Kleinschmidt, G. (Ed.): The Geology of the Antarctic Continent, Borntraeger, Stuttgart, 596 pp., 2021.

Melles, M. und Estrada, S.: Arbeitskreis Geologie und Geophysik der Polargebiete, in: 50 Jahre Deutsche Gesellschaft für Polarforschung e.V. 1959-2009, Herausgeber: Huch, M. und Tessensohn, F., Geoskript Agentur für Geowissenschaften + Öffentlichkeit, Bremerhaven, 2009.

Melles, M., Brigham-Grette, J., Minyuk, P. S., Nowaczyk, N. R., Wennrich, V., DeConto, R. M. Anderson, P. M., Andreev, A. A., Coletti, A., Cook, T. L., Haltia-Hovi, E., Kukkonen, M., Lozhkin, A. V., Rosén, P., Tarasov, P., Vogel, H., and Wagner, B.: 2.8 million years of Arctic climate change from Lake El'gygytgyn, NE Russia, Science, 337, 315-320, https://doi.org/10.1126/science.1222135, 2012.

Melles, M., Diekmann, B., Estrada, S., Gaedicke, C., Gohl, K., Jokat, W., Lembke-Jene, L., Läufer, A., Lisker, F., Piepjohn, K., Scheinert, M., Schirrmeister, L., Stein, R., Tessensohn, F., and Tiedemann, R.: Geowissenschaftliche Polarforschung in Deutschland - globale Bedeutung und Perspektive, Polarforschung, 85, 1-64, https://doi.org/10.2312/polarforschung.85.1.1, 2015.

Piepjohn, K., Strauss, J. V., Reinhardt, L., and McClelland, W. C. (Eds.): Circum-Arctic Structural Events: Tectonic Evolution of the Arctic Margins and Trans-Arctic Links with Adjacent Orogens, Boulder, Colorado, Geol. Soc. Am. Spec. Pap., 541, 1-8, https://doi.org/10.1130/2019.2541(01), 2019. 